\title{
Perceptions and practice of personal protective behaviors to prevent COVID-19 transmission in the G7 nations
}

\author{
Constantine I. Vardavas ${ }^{1}$, Satomi Odani ${ }^{1}$, Katerina Nikitara ${ }^{1}$, Hania El Banhawi ${ }^{1}$, Christina N. Kyriakos ${ }^{1}$, Luke Taylor ${ }^{2}$, Grace \\ Lown $^{2}$, Nicolas Becuwe ${ }^{3}$
}

\author{
AFFILIATION \\ 1 School of Medicine, University of Crete, Heraklion, Greece \\ 2 Kantar, Public Division, London, United Kingdom \\ 3 Kantar, Public Division, Brussels, Belgium
}

\section{CORRESPONDENCE TO}

Satomi Odani. School of Medicine, University of Crete, Heraklion, 715 00,

Crete, Greece. E-mail: odani@tobcontrol.eu
ORCID ID: https://orcid.org/0000-0002-6263-4952

KEYWORDS

COVID-19, health behavior, preventive medicine, infection control

Received: 18 May 2020, Accepted: 9 June 2020

https://doi.org/10.18332/popmed/123821

\begin{abstract}
INTRODUCTION To combat the transmission of COVID-19, countries have endorsed a series of non-pharmaceutical measures. We evaluated the practice and perceptions of personal protective measures and social distancing across the G7 countries.

METHODS Data were collected during 19-21 March 2020, from 7005 of Kantar's online panelists aged $>16$ years across the G7 countries: Canada, France, Great Britain, Germany, Italy, Japan, and the United States. Data were post-stratified and weighted to match population distributions of the respective countries. Descriptive and multivariable analyses were conducted in late March 2020.

RESULTS Males (vs females) and those less educated (vs college graduates) were less likely to practice personal protective measures and social distancing. Younger adults were also less likely to practice social distancing (vs adults
\end{abstract}

$>65$ years old). Respondents who expressed concern about the impact of COVID-19 on their health, income or education had higher odds of practicing personal protective measures (AOR=2.81, 1.74, and 1.54, respectively) and social distancing (AOR=3.18, 1.68, and 1.89, respectively) compared to those who did not. Those who perceived precautionary measures as highly effective were also more likely to practice personal protective measures $(\mathrm{AOR}=2.05)$ and social distancing $(A O R=3.99)$ compared to those who perceived them as ineffective.

CONCLUSIONS Concerns about COVID-19 and perceived effectiveness of precautionary measures strongly predict practice of protective measures, regardless of the types of behaviors. Population-wide interventions should focus on ensuring increased adherence and tailoring communications to groups that are less likely to practice protective behaviors.

\section{INTRODUCTION}

The COVID-19 respiratory infection pandemic has rapidly evolved into a global public health threat. By the 31 March 2020, about 0.78 million COVID-19 cases had been reported in over 190 countries, including 37272 deaths ${ }^{1}$. The acceleration of transmission is demonstrated by the fact that, while it took over three months for the number of confirmed cases to reach 10000, in only 12 days the next 100000 confirmed cases had already been reported ${ }^{2}$.

With the aim of minimizing transmission and the subsequent burden of COVID-19 to the healthcare system, countries have dedicated significant resources to preparedness and response strategies ${ }^{3}$. Governments have applied a series of predominantly non-pharmaceutical measures. These measures include the practice of personal protective measures (hand hygiene, respiratory etiquette, face masks), environmental measures (surface and object cleaning), social distancing (self-isolation, quarantine, school closures, workplace measures and closures), travel-related measures (travel advice, entry and exit screening, internal travel restrictions, border closures) as well as strategic risk communication and community engagement ${ }^{4}$.

The efficacy and impact of the aforementioned strategies are highly dependent on community compliance and 
cooperation $^{5}$. Encouraging and motivating people to comply with specific behaviors regarding hygiene and social distancing has previously proven effective in mitigating other infectious disease outbreaks ${ }^{6,7}$. Nevertheless, the adoption of such behaviors depends on many factors related to personal perceptions and beliefs about the effectiveness of the precautionary measures, the perceived risk of contracting the disease or having friends/family being affected by the disease, the perceived severity of the health, economic and educational consequences ${ }^{8-11}$.

A better understanding of people's behaviors, beliefs, concerns, knowledge, as well as of the associated predictive factors, during an emerging pandemic is of crucial importance for public health officials and decisionmakers, to enhance communication efforts for the promotion of individual and community health. Additionally, the cross-country exploration of differences in emergency preparedness and response strategies to a pandemic could provide useful information to identify effective approaches within the context of each country.

In light of the above and of the scarcity of relevant data for COVID-19, especially in the form of a cross-country comparison, this study aims to evaluate the public's perceptions and practice of personal protective measures and social distancing to prevent COVID-19 transmission across the G7 nations (Canada, Great Britain, France, Germany, Italy, Japan, United States) in late March 2020.

\section{METHODS}

Anonymous data were collected by the quota sampling method by the Public Division of Kantar between 19-21 March 2020, across all G7 countries: Canada, France, Great Britain (GB), Germany, Italy, Japan, and the US. Around 1000 online panelists, aged $>18$ years, responded per country, leading to a pooled sample size of 7005. Collected data were post-stratified with respect to gender by age group, and gender by degree-holding status within each country and were weighted using the US Census Bureau and education statistics from the Organization for Economic Co-operation and Development (OECD) to match population distributions for each of the G7 countries. All participants provided written informed consent to voluntarily participate in the study in which the data were fully anonymized. The current study was exempt from an ethics review by an institutional review board (IRB), as it was a secondary analysis of de-identified data.

\section{Measures}

The practice of protective behaviors

Protective behaviors were assessed by asking respondents: 'Some people are able to make changes to their lifestyle in response to the coronavirus outbreak. Since the start of the outbreak, have you started doing any the following?'. Respondents selected all that applied from ten provided items. Three aggregate categories were created: 1 ) personal protective measure (washing hands, using hand sanitizers, wearing a mask); 2) social distancing (working from home, self-isolating at home, avoiding non-essential social contact, avoiding handshakes/keeping a distance, avoiding public transportation, avoiding visits to the elderly and vulnerable relatives/friends, and avoiding visits to pubs/cafes/ restaurants); and 3) both personal protective and social distancing measures.

\section{Concerns about the impact of COVID-19 on health, income and} education

Concern about health was assessed using three questions that asked how much respondents were concerned about their health, the health of family and friends, and other people living in their country. Concern about community health was assessed using three questions, which asked how much respondents were concerned about health and wellbeing of their neighbors/community, availability of local health services, and local social care services for the elderly and vulnerable. Responses were dichotomized as concerned (very/fairly concerned) and not concerned (not very/not at all concerned, don't know).

Concern about income was assessed using two questions asking about respondents' personal income and household income. Respondents were classified as concerned if they answered: 'Coronavirus has already impacted on my personal/ household income' or 'Coronavirus has not yet impacted on my personal/household income, but I expect it to do so in the future' (vs not concerned, i.e. 'Coronavirus will have no impact on my personal/household income' or 'Don't know').

Concern about education was assessed with the question: 'If this is applicable to you, how concerned are you about either your own education or the education of your children?'. Respondents were classified as 'concerned (very/fairly concerned)', 'not concerned' (not very/not at all concerned or don't know), or not applicable.

Perceptions regarding precautionary measures and COVID-19 Perceived effectiveness of preventive measures was assessed using nine items: asking people to stay home, closing schools, closing colleges/universities, closing the country borders, shutting down public transport, closing public places, isolating older people, social distancing, and setting restrictions on movement outside the home. An index was created by summing up the number of items for which respondents provided an affirmative response (very/ fairly effective vs not very/not at all effective or don't know). The index score was classified into low $(0-3)$, moderate (4-6), and high (7-9). Perceived spread of COVID-19 was assessed by whether respondents themselves and/or their close family members/friends have contracted the virus. Responses were dichotomized into yes ('Yes, definitely', 'Yes, I think so', or 'Possibly') and no ('No', 'Don't know').

\section{Sociodemographic characteristics}

The following sociodemographic characteristics were 
assessed: country, gender, age, education attainment, presence of a child in the household, and the most trusted source of information on the COVID-19 outbreak.

\section{Statistical analyses}

Descriptive statistics were calculated for the G7 countries overall and by country with $95 \%$ confidence intervals (CIs). Chi-squared tests were used to examine within- and across-group differences with statistical significance set at $\mathrm{p}<0.05$. To investigate predictors of protective behaviors, multivariable logistic regression models were fitted separately for each of the three variables of protective behaviors (personal protective measures, social distancing, and both of these). Independent variables included in the models were selected from the variables that were associated with protective behaviors at the bivariate level, with the exception of the measure of concern about community health, which was not included in the final models as it was highly correlated with concern about health (Pearson correlation coefficient of 0.58 ). All analyses were conducted in late March 2020, using R version 3.6.2.

\section{RESULTS}

Concerns about the impact of COVID-19 on health, income and education

Table 1 shows the percentages of reported concerns about the impact of COVID-19 stratified by country and gender across the G7 countries. Overall, $90.8 \%$ of respondents reported any type of concern with regard to the impact of COVID-19 on health including their own health, the health of their families and friends, and the health of other people living in their country. In all, $89.1 \%$ of respondents reported any type of concern about the health and wellbeing of neighbors and community, availability of local health services and local social care services for the elderly and the vulnerable. In GB, Germany and the US, a higher percentage of females than males reported any concern about the impact of COVID-19 on local community's health and wellbeing, and the availability of services $(\mathrm{p}<0.05)$. Overall, $75.2 \%$ of respondents reported that COVID-19 had or is expected to impact their personal or household income (from $60.7 \%$ in Germany to $87.9 \%$ in Italy), and $41.7 \%$ reported concerns about the impact on their own or their children's education (from $31.5 \%$ in Germany to $44.3 \%$ in the US).

\section{Practice of protective behaviors}

Table 2 presents percentages of the practice of protective behaviors to prevent COVID-19 transmission by sociodemographic characteristics and relevant perceptions in G7 countries. Overall, 85.4\% of respondents reported practicing personal protective measures, $91.2 \%$ reported taking social distancing measures, and $81 \%$ reported taking both personal protective measures and social distancing measures. The most frequently reported personal protective measure was washing hands more often/for longer (76.5\%), followed by the use of hand sanitizing gel (53.9\%), and, to a lesser extent, the use of a mask (26.0\%). Among the social distancing measures, avoiding handshakes and keeping at a distance was most common (89.1\%). A smaller percentage reported working from home $(28.7 \%)$.

The practice of personal protective measures and social distancing measures is displayed by country in Figure 1. Country-specific breakdown by sociodemographics is presented in the Supplementary file (Table S1 for EU Countries and Table S2 for non-EU countries).

The practice of protective behaviors differed across sociodemographic groups. For the practice of both personal protective measures and social distancing, the percentage was higher in Italy (91.8\%), Canada (90.8\%) and France (86.8\%), and lower in Japan (61.8\%). The reported practice of both protective behaviors was higher among females $(85.8 \%)$ than males $(77.1 \%)$, older adults aged $\geq 65$ years $(86.5 \%)$ than young adults aged $16-24$ years $(74.7 \%)$, and those with a college/university degree $(86.0 \%)$ than those without any full-time education (72.5\%). By type of most trusted source of information, the percentage of practicing both protective behaviors was higher among those who reported doctors/healthcare providers (89.1\%), followed by government/politicians (88.1\%). Lower percentages were seen for those who reported social media as their trusted source of information $(78.3 \%$ ) (all $\mathrm{p}<0.05$ ).

Associations between concerns/perceptions towards the COVID-19 outbreak and practice of protective behaviors

Table 3 presents the associations between concerns and perceptions towards the COVID-19 outbreak and the practice of protective behaviors across the G7 nations. At the time of the survey, respondents from GB, Germany, Japan and the US, were less likely than respondents in Italy to practice personal protective behaviors and social distancing measures.

Males were less likely than females to practice both personal protective measures (AOR $=0.60 ; 95 \% \mathrm{CI}$ : 0.48-0.79) and social distancing measures (AOR $=0.59$; $95 \% \mathrm{CI}$ : $0.45-$ 0.79 ), while those with lower education were less likely to practice protective behaviors (AOR $=0.63$; 95\% CI: 0.43-0.89), social distancing (AOR $=0.45 ; 95 \% \mathrm{CI}: 0.27-0.75$ ), and both approaches (AOR $=0.54 ; 95 \%$ CI: 0.37-0.77). Older age was also positively associated with practicing social distancing, although a significant association was not observed between age and personal protective measures. The highest odds of practicing social distancing were observed among older adults $\geq 65$ years (AOR=3.09; 95\% CI: 1.61-5.96), followed by those aged $45-64$ years (AOR $=2.14 ; 95 \%$ CI: 1.16-3.96) and those aged $25-44$ years (AOR=1.99; 95\% CI: $1.11-3.56$ ) compared to those 16-24 years of age. Moreover, older adults aged $\geq 65$ years were more likely to practice both personal protective measures and social distancing $(\mathrm{AOR}=2.08 ; 95 \%$ CI: $1.32-3.26)$ compared to those aged $16-24$ years.

Respondents who expressed concern about the impact of COVID-19 on their health or the health of their family, friends 


\begin{tabular}{|c|c|c|c|c|c|c|c|c|c|c|c|c|}
\hline \multirow[t]{2}{*}{ Countries } & & & $\begin{array}{l}\text { Any concern } \\
\text { about health } \\
\text { - health of } \\
\text { my own, } \\
\text { family, } \\
\text { friends, and/ } \\
\text { or people in } \\
\text { the country }\end{array}$ & $\begin{array}{l}\text { My own } \\
\text { health }\end{array}$ & $\begin{array}{l}\text { Health of } \\
\text { family and } \\
\text { friends }\end{array}$ & $\begin{array}{l}\text { Health of } \\
\text { other people } \\
\text { living in the } \\
\text { country }\end{array}$ & $\begin{array}{l}\text { Any concern } \\
\text { about } \\
\text { community } \\
\text { health - } \\
\text { wellbeing } \\
\text { of the } \\
\text { community } \\
\text { and/or } \\
\text { availability } \\
\text { of services } \\
\end{array}$ & $\begin{array}{l}\text { Health and } \\
\text { wellbeing } \\
\text { of my } \\
\text { neighbors } \\
\text { and } \\
\text { community }\end{array}$ & $\begin{array}{c}\text { Availability } \\
\text { of local } \\
\text { health } \\
\text { services }\end{array}$ & $\begin{array}{l}\text { Local } \\
\text { social care } \\
\text { services for } \\
\text { the elderly } \\
\text { and the } \\
\text { vulnerable }\end{array}$ & $\begin{array}{l}\text { Concern - } \\
\text { impact on } \\
\text { personal/ } \\
\text { household } \\
\text { income }\end{array}$ & $\begin{array}{l}\text { Concern - } \\
\text { impact on } \\
\text { education }\end{array}$ \\
\hline & & $\mathbf{N}$ & $\%(95 \%$ CI $)$ & $\%(95 \% \mathrm{CI})$ & $\%(95 \%$ CI $)$ & $\%(95 \%$ CI $)$ & $\%(95 \% \mathrm{CI})$ & & & $\%(95 \% \mathrm{CI})$ & & $\%(95 \% \mathrm{CI})$ \\
\hline Overall & & 7005 & 90.8 (89.9-91.8) & $72.8(71.3-74.2)$ & $82.3(81.0-83.5)$ & $85.6(84.5-86.7)$ & 89.1 (88.1-90.1) & $77.3(76.0-78.6)$ & $77.6(76.3-79.0)$ & $81.4(80.2-82.6)$ & $75.2(73.8-76.6)$ & $41.7(40.1-43.3)$ \\
\hline \multirow[t]{3}{*}{ Canada } & Overall & 1000 & 93.5 (91.8-95.1) & $72.7(69.8-75.7)$ & 86.8 (84.6-89.1) & 89.8 (87.8-91.9) & 94.1 (92.4-95.7) & $84.0(81.5-86.5)$ & 84.1 (81.5-86.6) & $85.3(82.8-87.7)$ & 80.7 (77.9-83.4) & $40.9(37.7-44.2)$ \\
\hline & Female & 486 & $95.8(94.0-97.7)$ & 75.8 (71.8-79.9) & $89.5(86.5-92.4)$ & $93.0(90.5-95.4)$ & & $85.9(82.5-89.3)$ & & 87.8 (84.5-91.1) & $82.9(79.0-86.8)$ & $41.8(37.1-46.4)$ \\
\hline & Male & 507 & $91.3(88.6-94.0)$ & $69.5(65.2-73.8)$ & $84.3(80.9-87.8)$ & $86.8(83.7-90.0)$ & $92.5(90.0-95.1)$ & $81.8(78.1-85.5)$ & $81.4(77.7-85.1)$ & $82.9(79.3-86.5)$ & $78.5(74.6-82.5)$ & $39.7(35.2-44.2)$ \\
\hline \multirow[t]{3}{*}{ France } & Overall & 1000 & $93.6(91.9-95.4)$ & 76.1 (73.1-79.1) & 86.5 (84.1-88.9) & $87.7(85.4-90.0)$ & $93.0(91.1-94.9)$ & 83.1 (80.5-85.8) & $84.1(81.5-86.8)$ & 81.9 (79.1-84.7) & $70.6(67.3-74.0)$ & $43.1(39.6-46.5)$ \\
\hline & & 493 & & & & & & & & & & $43.3(38.4-48.2)$ \\
\hline & Male & 496 & & 73.4 (69.0-77.8) & & $85.3(81.6-88.9)$ & 91.8 (88.9-94.7) & & & 77.1 (72.8-81.3) & 72.7 (68.2-77.3) & $42.4(37.6-47.2)$ \\
\hline \multirow[t]{3}{*}{ GB } & Overall & 1001 & 93.4 (91.8-94.9) & $73.2(70.4-76.0)$ & 87.1 (85.0-89.2) & $86.6(84.4-88.7)$ & 93.4 (91.9-95.0) & 81.3 (78.8-83.7) & 84.1 (81.8-86.4) & $86.6(84.5-88.7)$ & 74.3 (71.5-77.2) & $40.4(37.3-43.5)$ \\
\hline & Female & 480 & $95.2(93.2-97.1)$ & $76.3(72.3-80.3)$ & 89.9 (87.1-92.7) & 88.6 (85.7-91.6) & $96.6(94.9-98.2)$ & $86.7(83.6-89.8)$ & $89.3(86.4-92.1)$ & $91.3(88.8-93.9)$ & 75.9 (71.8-80.1) & $43.2(38.5-47.8)$ \\
\hline & Male & 514 & $91.6(89.2-94.1)$ & $70.6(66.6-74.6)$ & $84.8(81.7-87.9)$ & 84.8 (81.6-87.9) & $90.4(87.8-93.0)$ & $76.3(72.6-80.0)$ & $79.3(75.7-82.9)$ & $82.1(78.7-85.4)$ & & $37.5(33.3-41.7)$ \\
\hline \multirow[t]{3}{*}{ Germany } & Overall & 1004 & 90.3 (88.4-92.2) & 68.4 (65.4-71.4) & & & & & & 75.7 (72.9-78.5) & & $31.5(28.5-34.5)$ \\
\hline & Female & 482 & $92.6(90.2-95.0)$ & 71.0 (66.6-75.3) & $81.6(77.9-85.3)$ & $85.3(82.0-88.6)$ & $91.0(88.3-93.7)$ & $75.1(71.0-79.2)$ & $72.3(67.9-76.7)$ & $80.5(76.7-84.4)$ & 58.7 (53.8-63.7) & $33.5(28.9-38.0)$ \\
\hline & Male & 515 & $88.4(85.6-91.2)$ & 65.9 (61.7-70.1) & 74.4 (70.5-78.3) & $78.6(75.0-82.2)$ & $85.1(82.0-88.3)$ & $68.0(63.9-72.2)$ & $62.4(58.2-66.7)$ & $71.4(67.4-75.4)$ & 63.4 (59.1-67.8) & $29.4(25.4-33.4)$ \\
\hline \multirow[t]{3}{*}{ Italy } & Overall & 1000 & 97.4 (96.4-98.5) & 85.3 (82.8-87.8) & 93.0 (91.2-94.8) & 93.9 (92.3-95.5) & 96.2 (94.7-97.6) & 89.8 (87.7-91.9) & 90.5 (88.4-92.7) & 92.1 (90.1-94.1) & 87.9 (85.6-90.3) & $41.4(37.9-44.8)$ \\
\hline & Female & 479 & 98.3 (97.3-99.3) & 86.9 (83.5-90.4) & & $95.3(93.3-97.4)$ & & & & $92.2(89.2-95.1)$ & $90.3(87.1-93.5)$ & $43.1(38.0-48.2)$ \\
\hline & Male & 511 & $97.5(96.0-99.0)$ & 84.4 (80.9-87.9) & 93.1 (90.7-95.6) & 93.7 (91.4-95.9) & 97.0 (95.3-98.6) & 88.4 (85.4-91.5) & 89.8 (86.8-92.8) & 93.1 (90.7-95.6) & $85.3(81.7-88.8)$ & $39.3(34.6-43.9)$ \\
\hline \multirow[t]{3}{*}{ Japan } & Overall & 1000 & 82.7 (79.9-85.4) & 65.1 (61.5-68.6) & $69.1(65.7-72.6)$ & 78.1 (75.1-81.1) & 73.5 (70.2-76.8) & $55.8(52.1-59.5)$ & $60.0(56.4-63.7)$ & $66.0(62.5-69.5)$ & $69.7(66.2-73.1)$ & $42.3(38.6-45.9)$ \\
\hline & Female & 498 & $85.9(82.2-89.6)$ & $65.1(59.9-70.3)$ & 72.7 (67.8-77.6) & 81.0 (76.8-85.1) & 74.8 (70.1-79.6) & & $62.0(56.7-67.3)$ & 66.4 (61.3-71.5) & 70.1 (65.1-75.1) & $41.5(36.1-46.8)$ \\
\hline & Male & 480 & $81.1(77.2-85.1)$ & $66.1(61.2-71.0)$ & $67.7(62.9-72.6)$ & $77.8(73.6-82.0)$ & 73.8 (69.4-78.3) & $56.7(51.6-61.9)$ & $59.1(54.0-64.3)$ & $67.4(62.6-72.2)$ & $70.8(66.0-75.6)$ & $43.9(38.7-49.1)$ \\
\hline \multirow[t]{3}{*}{ US } & Overall & 1000 & $91.6(89.9-93.4)$ & 73.8 (71.0-76.6) & $84.2(81.9-86.5)$ & 86.9 (84.8-89.1) & $92.2(90.5-93.9)$ & 82.3 (79.9-84.7) & $81.5(79.0-84.0)$ & $85.4(83.2-87.7)$ & 79.1 (76.5-81.7) & $44.3(41.1-47.4)$ \\
\hline & Female & 497 & $94.4(92.4-96.4)$ & $78.2(74.5-81.9)$ & $88.4(85.5-91.2)$ & $90.7(88.1-93.3)$ & $95.7(93.9-97.5)$ & $87.3(84.4-90.2)$ & $87.9(84.8-90.9)$ & $90.6(87.9-93.3)$ & $83.2(79.6-86.7)$ & $45.1(40.7-49.6)$ \\
\hline & Male & 491 & $89.2(86.3-92.0)$ & $69.6(65.4-73.8)$ & $80.4(76.8-84.0)$ & $83.3(79.8-86.7)$ & $89.2(86.3-92.1)$ & $77.8(74.0-81.6)$ & $75.2(71.2-79.1)$ & $80.6(77.0-84.2)$ & $75.2(71.2-79.1)$ & $43.6(39.1-48.1)$ \\
\hline
\end{tabular}

Across-country differences are significant $(\mathrm{p}<0.05)$ for all items, among both males and females. Bold type indicates that differences are significant within the country by gender $(\mathrm{p}<0.05)$. 
Table 2. Practice of protective behaviors to prevent COVID-19 transmission, by sociodemographic characteristics, relevant concerns and perceptions in G7 countries, late March 2020

\begin{tabular}{|c|c|c|c|c|}
\hline \multirow[t]{2}{*}{$\begin{array}{l}\text { Characteristics } \\
\text { Concerns } \\
\text { Perceptions }\end{array}$} & & $\begin{array}{l}\text { Personal } \\
\text { protective } \\
\text { measures }\end{array}$ & Social distancing & $\begin{array}{c}\text { Both personal } \\
\text { protective } \& \\
\text { social distancing } \\
\text { measures }\end{array}$ \\
\hline & $\mathbf{N}$ & $\%(95 \% \mathrm{CI})$ & $\%(95 \% \mathrm{CI})$ & $\%(95 \% \mathrm{CI})$ \\
\hline Overall & 7005 & $85.4(84.3-86.6)$ & $91.2(90.3-92.1)$ & $81.0(79.8-82.3)$ \\
\hline \multicolumn{5}{|l|}{ Country } \\
\hline Canada & 1000 & $92.1(90.4-93.9)$ & $96.8(95.6-98.0)$ & 90.8 (88.9-92.7) \\
\hline France & 1000 & $88.1(86.0-90.3)$ & $96.3(95.0-97.5)$ & $86.8(84.5-89.1)$ \\
\hline GB & 1001 & $85.4(83.2-87.6)$ & $92.3(90.7-94.0)$ & 81.5 (79.1-83.9) \\
\hline Germany & 1004 & $81.4(78.9-83.9)$ & $93.5(91.8-95.1)$ & $79.2(76.6-81.8)$ \\
\hline Italy & 1000 & $92.4(90.6-94.2)$ & $98.1(97.2-99.0)$ & 91.8 (90.0-93.7) \\
\hline Japan & 1000 & 78.7 (75.7-81.8) & $73.8(70.6-77.1)$ & $61.8(58.2-65.4)$ \\
\hline US & 1000 & 86.5 (84.4-88.7) & 94.3 (92.9-95.8) & $84.8(82.5-87.0)$ \\
\hline \multicolumn{5}{|l|}{ Gender } \\
\hline Female & 3415 & $89.9(88.5-91.2)$ & $93.3(92.2-94.4)$ & $85.8(84.3-87.3)$ \\
\hline Male & 3514 & $82.0(80.2-83.7)$ & 89.5 (88.1-90.9) & $77.1(75.2-79.0)$ \\
\hline \multicolumn{5}{|l|}{ Age (years) } \\
\hline $16-24$ & 810 & $81.9(78.3-85.6)$ & $86.7(83.3-90.0)$ & $74.7(70.5-78.8)$ \\
\hline $25-44$ & 2592 & $80.8(78.7-83.0)$ & 90.3 (88.7-91.8) & $76.8(74.6-79.0)$ \\
\hline $45-64$ & 2584 & $88.2(86.4-90.0)$ & $91.9(90.6-93.3)$ & $83.4(81.4-85.4)$ \\
\hline$\geq 65$ & 1019 & $89.4(87.2-91.6)$ & $93.6(91.8-95.4)$ & $86.5(84.0-88.9)$ \\
\hline \multicolumn{5}{|l|}{ Education } \\
\hline No full-time education & 571 & $79.2(74.9-83.6)$ & $85.8(82.1-89.5)$ & $72.5(67.8-77.3)$ \\
\hline Still studying & 814 & $82.0(78.5-85.5)$ & $91.2(88.7-93.8)$ & $76.3(72.4-80.2)$ \\
\hline$<$ College/University & 3242 & $88.3(86.8-89.8)$ & $91.8(90.5-93.0)$ & $84.0(82.3-85.7)$ \\
\hline$\geq$ College/University & 1896 & $88.7(86.7-90.7)$ & $94.8(93.5-96.1)$ & $86.0(83.9-88.1)$ \\
\hline \multicolumn{5}{|l|}{ Presence of a child in the household } \\
\hline No & 4153 & $85.3(83.9-86.8)$ & $91.2(90.1-92.4)$ & $81.0(79.4-82.5)$ \\
\hline Yes & 2852 & $85.6(83.8-87.4)$ & $91.1(89.6-92.5)$ & $81.2(79.2-83.2)$ \\
\hline \multicolumn{5}{|l|}{ Concern - impact on health } \\
\hline No at all/not very concerned, don't know & 582 & $56.2(50.9-61.5)$ & $63.8(58.7-68.8)$ & $45.4(40.0-50.7)$ \\
\hline Fairly/very concerned & 6423 & $88.4(87.3-89.5)$ & $93.9(93.1-94.7)$ & $84.6(83.4-85.8)$ \\
\hline \multicolumn{5}{|l|}{ Concern - impact on the community } \\
\hline No at all/not very concerned, don't know & 679 & $57.2(52.4-62.0)$ & $62.3(57.6-67.0)$ & $43.9(39.1-48.8)$ \\
\hline Fairly/very concerned & 6326 & $88.9(87.8-90.0)$ & $94.7(94.0-95.4)$ & $85.6(84.4-86.7)$ \\
\hline \multicolumn{5}{|l|}{ Concern - impact on finances } \\
\hline Will have no impact & 1612 & $78.8(76.1-81.4)$ & $84.2(81.8-86.5)$ & $72.9(70.0-75.7)$ \\
\hline Already impacted/expect to impact in the future & 5393 & $87.6(86.4-88.8)$ & $93.5(92.6-94.4)$ & $83.7(82.4-85.1)$ \\
\hline \multicolumn{5}{|l|}{ Concern - impact on education } \\
\hline No at all/not very concerned, don't know & 1610 & $75.9(73.0-78.7)$ & $84.1(81.7-86.5)$ & $68.2(65.1-71.3)$ \\
\hline Fairly/very concerned & 2985 & $88.7(87.2-90.3)$ & $94.6(93.5-95.7)$ & $85.2(83.5-86.9)$ \\
\hline Not applicable & 2410 & $87.8(86.0-89.6)$ & $91.7(90.3-93.2)$ & $84.5(82.5-86.4)$ \\
\hline
\end{tabular}


Table 2. Continued

\begin{tabular}{|c|c|c|c|c|}
\hline \multirow[t]{2}{*}{$\begin{array}{l}\text { Characteristics } \\
\text { Concerns } \\
\text { Perceptions }\end{array}$} & & $\begin{array}{l}\text { Personal } \\
\text { protective } \\
\text { measures }\end{array}$ & Social distancing & $\begin{array}{c}\text { Both personal } \\
\text { protective } \& \\
\text { social distancing } \\
\text { measures }\end{array}$ \\
\hline & $\mathbf{N}$ & $\%(95 \% \mathrm{CI})$ & $\%(95 \% \mathrm{CI})$ & $\%(95 \% \mathrm{CI})$ \\
\hline \multicolumn{5}{|c|}{ Perceived effectiveness of precaution } \\
\hline Low (score $0-3$ ) & 502 & $55.6(50.1-61.2)$ & $56.2(50.6-61.7)$ & $39.7(34.3-45.2)$ \\
\hline Moderate (score 4-6) & 859 & $80.5(77.1-83.8)$ & $84.0(80.9-87.2)$ & $70.3(66.4-74.2)$ \\
\hline High (score 7-9) & 5644 & $89.1(88.0-90.3)$ & $95.7(95.0-96.4)$ & $86.7(85.5-88.0)$ \\
\hline \multicolumn{5}{|c|}{$\begin{array}{l}\text { Perceived spread of the infection - self/family/ } \\
\text { friends }\end{array}$} \\
\hline No/don't know & 5608 & $86.6(85.4-87.8)$ & $91.0(90.0-92.0)$ & $82.2(80.8-83.5)$ \\
\hline Yes & 1397 & $79.7(76.7-82.6)$ & $92.1(90.1-94.1)$ & $75.2(72.0-78.4)$ \\
\hline \multicolumn{5}{|c|}{ Most trusted information source } \\
\hline Doctors/healthcare providers & 1403 & $90.2(88.0-92.3)$ & $97.2(96.0-98.4)$ & $89.1(86.9-91.3)$ \\
\hline Friends/family & 353 & $76.1(70.1-82.2)$ & $90.9(87.5-94.3)$ & $70.0(63.6-76.4)$ \\
\hline Government/politicians & 1183 & $90.7(88.4-92.9)$ & $96.1(94.7-97.5)$ & $88.1(85.6-90.6)$ \\
\hline Newspapers/websites & 622 & $86.5(83.1-90.0)$ & $91.0(87.9-94.2)$ & $81.0(76.9-85.1)$ \\
\hline Social media & 358 & $81.4(75.8-87.0)$ & $94.3(91.1-97.6)$ & $78.3(72.5-84.2)$ \\
\hline TV news & 2221 & 87.8 (85.9-89.7) & $91.5(89.9-93.0)$ & $82.4(80.3-84.6)$ \\
\hline Other & 230 & $77.9(70.4-85.3)$ & $89.5(84.2-94.9)$ & $69.4(61.2-77.6)$ \\
\hline None of the above & 313 & $69.9(63.1-76.7)$ & $71.5(64.9-78.1)$ & $61.5(54.3-68.8)$ \\
\hline Don't know & 322 & $68.6(62.1-75.0)$ & $69.4(63.0-75.8)$ & $59.5(52.7-66.4)$ \\
\hline
\end{tabular}

Distributions were significantly different $(\mathrm{p}<0.05)$ within all population subgroups except presence of a child (personal protective behaviors, social distancing, and both personal protective \& social distancing behaviors) and perceived spread of the infection (social distancing).

Figure 1. The use of both personal protective and social distancing measures by country, late March 2020 $(\mathrm{N}=7005)$
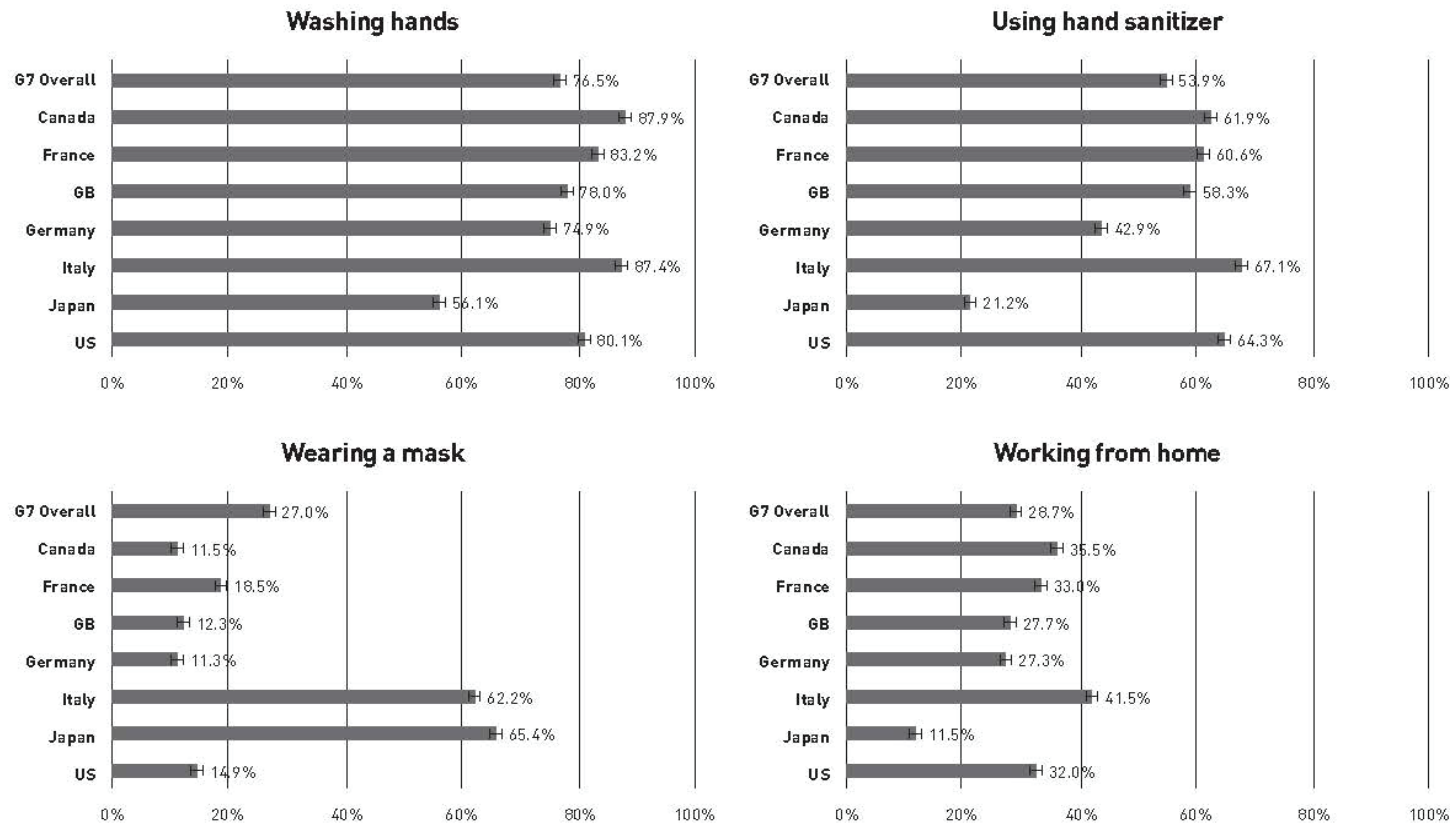
Figure 1. Continued
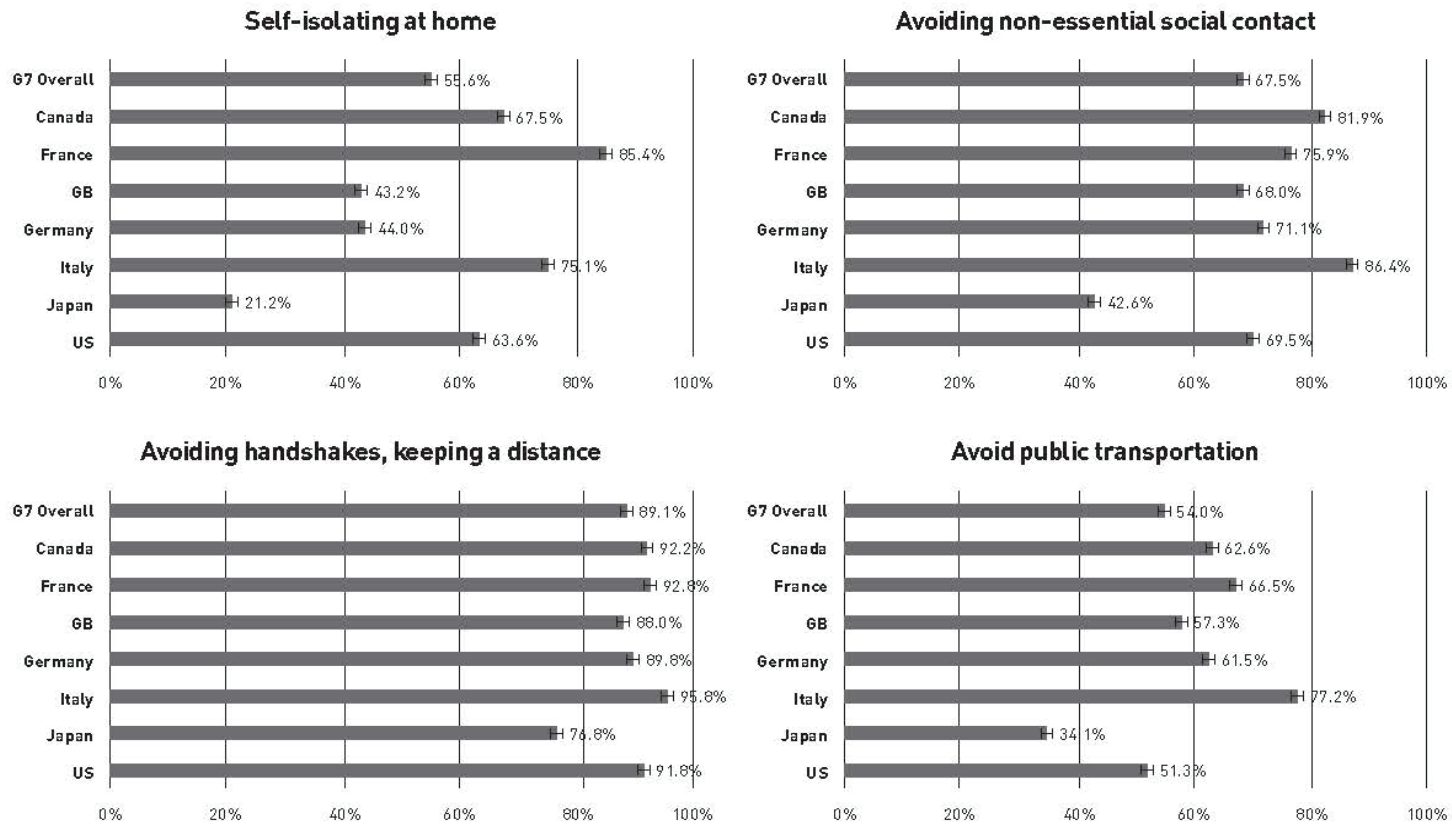

Avoid visits to the elderly/vulnerable

Avoid visits to pubs, cafes and restaurants
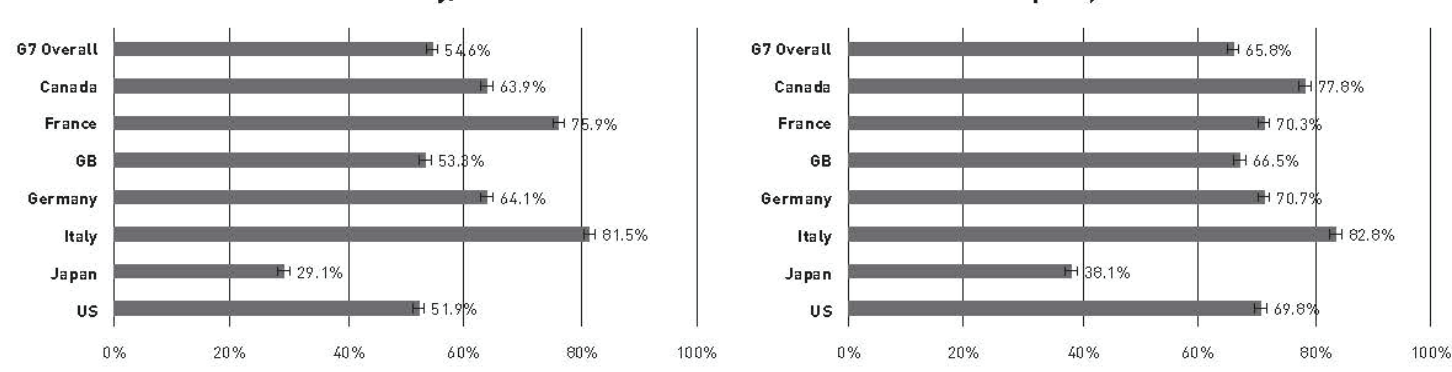

Table 3. Associations between sociodemographic characteristics, concerns and perceptions and the practice of protective behaviors to prevent COVID-19 transmission in G7 countries, late March 2020 (N=7005)

\begin{tabular}{|c|c|c|c|c|}
\hline \multirow[t]{2}{*}{$\begin{array}{l}\text { Characteristics } \\
\text { Concerns } \\
\text { Perceptions }\end{array}$} & \multirow[t]{2}{*}{ Categories } & $\begin{array}{l}\text { Personal } \\
\text { protective } \\
\text { measures }\end{array}$ & Social distancing & $\begin{array}{c}\text { Both personal } \\
\text { protective } \& \\
\text { social distancing } \\
\text { measures }\end{array}$ \\
\hline & & AOR $(95 \% \mathrm{CI})$ & AOR $(95 \% \mathrm{CI})$ & AOR (95\% CI) \\
\hline \multirow[t]{7}{*}{ Country } & Italy & Ref. & Ref. & Ref. \\
\hline & France & $0.80(0.55-1.16)$ & 0.58 (0.28-1.19) & $0.76(0.53-1.09)$ \\
\hline & GB & $0.58(0.41-0.83)$ & $0.25(0.13-0.47)$ & $0.47(0.34-0.67)$ \\
\hline & Germany & $0.48(0.34-0.67)$ & $0.40(0.21-0.77)$ & $0.46(0.33-0.65)$ \\
\hline & Canada & $1.05(0.71-1.55)$ & $0.49(0.24-1.01)$ & $0.95(0.65-1.38)$ \\
\hline & Japan & $0.63(0.42-0.93)$ & $0.09(0.05-0.17)$ & $0.25(0.18-0.36)$ \\
\hline & US & $0.70(0.49-1.01)$ & $0.38(0.19-0.74)$ & $0.63(0.44-0.90)$ \\
\hline \multirow[t]{2}{*}{ Gender } & Female & Ref. & Ref. & Ref. \\
\hline & Male & $0.60(0.48-0.74)$ & $0.59(0.45-0.79)$ & $0.61(0.50-0.74)$ \\
\hline
\end{tabular}


Table 3. Continued

\begin{tabular}{|c|c|c|c|c|}
\hline \multirow[t]{2}{*}{$\begin{array}{l}\text { Characteristics } \\
\text { Concerns } \\
\text { Perceptions }\end{array}$} & \multirow[t]{2}{*}{ Categories } & $\begin{array}{l}\text { Personal } \\
\text { protective } \\
\text { measures }\end{array}$ & Social distancing & $\begin{array}{c}\text { Both personal } \\
\text { protective } \& \\
\text { social distancing } \\
\text { measures } \\
\end{array}$ \\
\hline & & AOR $(95 \% \mathrm{CI})$ & AOR $(95 \% \mathrm{CI})$ & AOR (95\% CI) \\
\hline \multirow[t]{4}{*}{ Age (years) } & $16-24$ & Ref. & Ref. & Ref. \\
\hline & $25-44$ & $0.76(0.49-1.15)$ & $1.99(1.11-3.56)$ & $1.02(0.69-1.50)$ \\
\hline & $45-64$ & $1.24(0.78-1.98)$ & $2.14(1.16-3.96)$ & $1.41(0.93-2.13)$ \\
\hline & $\geq 65$ & $1.42(0.86-2.33)$ & $3.09(1.61-5.96)$ & $2.08(1.32-3.26)$ \\
\hline \multirow[t]{4}{*}{ Education } & No full-time education & $0.63(0.43-0.92)$ & $0.45(0.27-0.75)$ & $0.54(0.37-0.77)$ \\
\hline & Still studying & $0.77(0.50-1.19)$ & $0.81(0.43-1.50)$ & $0.67(0.45-1.01)$ \\
\hline & $<$ College/University & $1.05(0.80-1.36)$ & $0.62(0.43-0.89)$ & $0.88(0.69-1.12)$ \\
\hline & $\geq$ College/University & Ref. & Ref. & Ref. \\
\hline \multirow{2}{*}{$\begin{array}{l}\text { Concern - impact on health } \\
\text { (yourself, family, friends, } \\
\text { and/or people in the } \\
\text { country) }\end{array}$} & $\begin{array}{l}\text { No at all/not very } \\
\text { concerned, don't know }\end{array}$ & Ref. & Ref. & Ref. \\
\hline & Fairly/very concerned & $2.81(2.02-3.93)$ & $3.18(2.21-4.56)$ & $2.87(2.08-3.97)$ \\
\hline \multirow{2}{*}{$\begin{array}{l}\text { Concern - impact on } \\
\text { finances }\end{array}$} & Will have no impact & Ref. & Ref. & Ref. \\
\hline & $\begin{array}{l}\text { Already impacted/expect to } \\
\text { impact in the future }\end{array}$ & $1.54(1.20-1.98)$ & $1.89(1.38-2.58)$ & $1.65(1.31-2.08)$ \\
\hline \multirow[t]{3}{*}{$\begin{array}{l}\text { Concern - impact on } \\
\text { education }\end{array}$} & $\begin{array}{l}\text { No at all/not very } \\
\text { concerned, don't know }\end{array}$ & Ref. & Ref. & Ref. \\
\hline & Fairly/very concerned & $1.74(1.32-2.28)$ & $1.68(1.15-2.45)$ & $1.78(1.39-2.29)$ \\
\hline & Not applicable & $1.52(1.15-2.00)$ & $1.15(0.8-1.66)$ & $1.48(1.14-1.92)$ \\
\hline \multirow{3}{*}{$\begin{array}{l}\text { Perceived effectiveness of } \\
\text { precaution }\end{array}$} & Low (score 0-3) & Ref. & Ref. & Ref. \\
\hline & Moderate (score 4-6) & $1.49(1.02-2.19)$ & $1.99(1.31-3.01)$ & $1.67(1.16-2.41)$ \\
\hline & High (score 7-9) & $2.05(1.43-2.95)$ & $3.99(2.69-5.92)$ & $2.82(2.01-3.96)$ \\
\hline \multirow{2}{*}{$\begin{array}{l}\text { Perceived spread - self/ } \\
\text { family/friends }\end{array}$} & No/don't know & Ref. & Ref. & Ref. \\
\hline & Yes & $0.57(0.44-0.73)$ & $0.70(0.46-1.06)$ & $0.57(0.44-0.72)$ \\
\hline \multirow[t]{9}{*}{$\begin{array}{l}\text { Most trusted information } \\
\text { source }\end{array}$} & $\begin{array}{l}\text { Doctors/healthcare } \\
\text { providers }\end{array}$ & Ref. & Ref. & Ref. \\
\hline & Friends/family & $0.44(0.28-0.70)$ & $0.50(0.24-1.01)$ & $0.42(0.27-0.67)$ \\
\hline & Government/politicians & $0.90(0.60-1.35)$ & $0.58(0.31-1.07)$ & $0.77(0.53-1.12)$ \\
\hline & Newspapers/websites & $0.59(0.38-0.90)$ & $0.30(0.15-0.59)$ & $0.50(0.34-0.74)$ \\
\hline & Social media & $0.54(0.32-0.89)$ & $0.58(0.23-1.46)$ & $0.55(0.33-0.90)$ \\
\hline & TV news & $0.71(0.50-0.99)$ & $0.38(0.23-0.63)$ & $0.63(0.46-0.86)$ \\
\hline & Other & $0.60(0.32-1.12)$ & $0.41(0.16-1.03)$ & $0.46(0.25-0.83)$ \\
\hline & None of the above & $0.44(0.27-0.71)$ & $0.17(0.09-0.33)$ & $0.41(0.25-0.66)$ \\
\hline & Don't know & $0.43(0.27-0.67)$ & $0.17(0.09-0.32)$ & $0.38(0.25-0.59)$ \\
\hline
\end{tabular}


and people in their country had nearly three times higher odds of practicing personal protective measures $(\mathrm{AOR}=2.81$; 95\% CI: 2.02-3.93) and social distancing (AOR=3.18; 95\% CI: 2.21-4.56). Similarly, those who were concerned about the impact of COVID-19 on their own or their children's education were more likely to practice personal protective measures (AOR=1.74; 95\% CI: 1.32-2.28) and social distancing (AOR=1.68; 95\% CI: 1.15-2.45). Moreover, respondents who were concerned about the impact of COVID-19 on their income were more likely to practice protective behaviors (AOR=1.54, 1.89, and 1.65 for personal protective measures, social distancing, or both, respectively).

Perceived effectiveness of precautionary measures was also associated with the practice of protective behaviors: the odds of practicing social distancing were 3.99 (95\% CI: 2.69-5.92) times higher among those who had a high level of perceived effectiveness compared to those with a low level of perceived effectiveness. Respondents who reported that they or a close family member/friend had contracted COVID-19 had a lower likelihood of practicing personal protective measures and practicing both approaches $(\mathrm{AOR}=0.57$ in both cases). Finally, compared to respondents who reported doctors or healthcare providers as their most trusted source of information, those who reported any other type of information source (with the exception of government/ politicians) were significantly less likely to practice both protective behaviors.

\section{DISCUSSION}

This is one of the first studies to assess public perceptions and behaviors during the COVID-19 pandemic using data from seven countries. Among G7 countries, over 75\% of respondents reported washing hands more often/for longer since the start of the outbreak. While slightly different measures, this compares to the Wang et al. ${ }^{12}(2020)$ study in which in the past 14 days, $66.6 \%$ reported always washing hands after touching contaminated objects. In both studies, among all protective measures, washing hands was the most frequently reported, which may be a result of the emphasis placed by the World Health Organization on handwashing as the official guidance for how to protect yourself from COVID-1912. Almost all Ministries of Health have also published guidelines on how to wash hands properly. Hand-washing is also the easiest measure to practice, while sanitizing products and masks are more dependent on product availability.

With regard to masks, at the time of the survey there was controversy on whether they should be worn by the general public, and personal discomfort and sense of embarrassment could also affect compliance ${ }^{13}$. In the present study, handwashing was more commonly practiced than wearing a mask in all G7 countries except Japan, where wearing a mask was more frequently reported (65\%) than washing hands (56\%). It is reasonable that Japan had the highest percentage of reported mask use given that it is a common practice in
Japan for the prevention of sickness and as general etiquette. After Japan, Italy also had 2.3-5.4 times higher reported mask use (62\%, just slightly lower than Japan) than other G7 countries. This could be explained by differences in the stage of the outbreak at the time of data collection in each of the G7 countries, demonstrated by the varied numbers of confirmed cases and fatalities. For instance, during the fieldwork dates, the number of cases in Italy had reached 53578, while less than half that number had been reported in Germany (22364 cases $)^{14}$. Other possible explanations include differences in governmental responses to the outbreak, risk communication messages and guidelines on personal protective measures across countries.

Concern about COVID-19 was a strong predictor of practicing protective behaviors regardless of the type of behavior (personal protective measures or social distancing). Of the three aspects assessed (health, income, and education), concern about health showed the strongest association. On average, $82 \%$ of respondents reported being concerned about the health of family and friends. These results compare to a population-based survey in China by Wang et al. ${ }^{12}$ (2020), in which $75.2 \%$ of respondents reported being very or somewhat worried about family members getting COVID-19. Notably, in our study, more respondents were concerned about the health and wellbeing of other people living in their country and their local communities than for their own health. This suggests that focus should be placed on conveying how protective measures are vital to safeguarding the health and wellbeing of others.

Our findings revealed a significant association between perceived effectiveness of precautionary measures and practicing protective measures. It is critical to inform the public of the purposes and expected effects of recommended preventive measures to keep them in compliance and to promote personal protective behaviors. Furthermore, compared to other information sources, people who most trusted doctors/healthcare providers and government/ politicians were more likely to practice protective behaviors, highlighting the importance of their roles in health communication. Consistent with previous findings ${ }^{15,16}$, our study revealed disparities in protective behaviors as demonstrated by the lower practice among males, young people, and those with less education. Health communication strategies should utilize data and various channels to maximize the reach of the messages. Attention should also be made to message framing to avoid confusion and engage all populations, including those with lower health literacy and the vulnerable ${ }^{17}$.

\section{Limitations}

Our study has several limitations. First, by using online access panels as the sample source, where people self-select onto these panels, there is the possibility of residual bias not addressed by post-stratification and weighting. Second, due to the cross-sectional nature of the study, we cannot establish 
causal relationships. Finally, we were unable to consider any possible effects of ecological factors that could strongly predict people's behaviors (e.g. differences in social damages from the COVID-19 pandemic, instructions or laws adopted in each country, cultural differences, social norms).

\section{CONCLUSIONS}

Our findings detail the picture of the perceptions and practice of personal protective behaviors across the G7 nations during the COVID-19 pandemic. The majority of respondents were concerned about the impact of the COVID-19 pandemic on health, income, and education, with respondents who expressed these concerns more likely to practice protective measures. Personal behaviors to prevent and reduce the spread of the virus are the frontline measures to control this pandemic. Hence, population-wide interventions should focus on ensuring increased adherence and tailoring health-related communications to groups that are less likely to practice protective measures.

\section{REFERENCES}

1. European Centre for Disease Prevention and Control. Situation update worldwide, as of 31 March 2020, 08:00. European Centre for Disease Prevention and Control. https:// www.ecdc.europa.eu/en/geographical-distribution-2019ncov-cases. Published March 31, 2020. Accessed April 6, 2020.

2. World Health Organization. Situation Report-59. https:// www.who.int/docs/default-source/coronaviruse/situationreports/20200319-sitrep-59-covid-19.pdf?sfvrsn=c3dcdef9_2. Published March 19, 2020. Accessed April 6, 2020.

3. World Health Organization. Strategic Preparedness and Response Plan. https://www.who.int/docs/default-source/ coronaviruse/srp-04022020.pdf. Published March 19, 2020. Accessed April 6, 2020.

4. World Health Organization. Non-pharmaceutical public health measures for mitigating the risk and impact of epidemic and pandemic influenza. https://apps.who.int/iris/ bitstream/handle/10665/329438/9789241516839-eng. pdf?ua=1. Published October 2019. Accessed April 6, 2020.

5. Low DE. Pandemic planning: Non-pharmaceutical interventions. Respirology. 2008;13(1):S44-S48. doi:10.1111/j.1440-1843.2008.01258.x

6. Fung ICH, Cairncross S. Effectiveness of handwashing in preventing SARS: a review. Tropical Medicine \& International Health. 2006;11(11):1749-1758. doi:10.1111/j.1365-3156.2006.01734.x

7. Fong MW, Gao H, Wong JY, et al. Nonpharmaceutical measures for pandemic influenza in nonhealthcare settingssocial distancing measures. Emerging Infectious Diseases.

\section{CONFLICTS OF INTEREST}

The authors have completed and submitted the ICMJE Form for Disclosure of Potential Conflicts of Interest and none was reported.

FUNDING

There was no source of funding for this research.

\section{0;26(5):976-984. doi:10.3201/eid2605.190995}

8. Lau JTF, Yang X, Tsui H, Kim JH. Monitoring community responses to the SARS epidemic in Hong Kong: from day 10 to day 62. Journal of Epidemiology \& Community Health. 2003;57(11):864-870. doi:10.1136/jech.57.11.864

9. Tang CS, Wong C. Factors influencing the wearing of facemasks to prevent the severe acute respiratory syndrome among adult Chinese in Hong Kong. Preventive Medicine. 2004;39(6):1187-1193. doi:10.1016/j.ypmed.2004.04.032

10.Tang CSK, Wong C. An outbreak of the severe acute respiratory syndrome: predictors of health behaviors and effect of community prevention measures in Hong Kong, China. Am J Public Health. 2003;93(11):1887-1888. doi:10.2105/ajph.93.11.1887

11. Leung GM, Quah S, Ho LM, et al. A tale of two cities: community psychobehavioral surveillance and related impact on outbreak control in Hong Kong and Singapore during the severe acute respiratory syndrome epidemic. Infection Control \& Hospital Epidemiology. 2004;25(12):1033-1041. doi:10.1086/502340

12. Wang C, Pan R, Wan X, et al. Immediate psychological responses and associated factors during the initial stage of the 2019 coronavirus disease (COVID-19) epidemic among the general population in China. International Journal of Environmental Research and Public Health. 2020;17(5):1729. doi:10.3390/ijerph17051729

13. Sim SW, Moey KSP, Tan NC. The use of facemasks to prevent respiratory infection: a literature review in the context of the Health Belief Model. Singapore Medical Journal. 2014;55(3):160. doi:10.11622/smedj.2014037

14. Worldometer. Coronavirus update (live). Worldometer https://www.worldometers.info/coronavirus/\#countries. Published 2020. Accessed March 31, 2020.

15.Zhong BL, Luo W, Li HM, et al. Knowledge, attitudes, and practices towards COVID-19 among Chinese residents during the rapid rise period of the COVID-19 outbreak: a quick online cross-sectional survey. International Journal of Biological Sciences. 2020;16(10):1745-1752. doi:10.7150/ijbs.45221

16. Grantz KH, Rane MS, Salje H, Glass GE, Schachterle SE, Cummings DAT. Disparities in influenza mortality and transmission related to sociodemographic factors within Chicago in the pandemic of 1918. Proceedings of the National Academy of Sciences. 2016;113(48):13839-13844. doi:10.1073/pnas.1612838113

17.Zarcadoolas C, Pleasant A, Greer DS. Advancing health literacy: A framework for understanding and action. Vol 45. Hoboken, NJ: John Wiley \& Sons; 2009.

AUTHORS' CONTRIBUTIONS

All authors contributed significantly to this work.

PROVENANCE AND PEER REVIEW

Not commissioned; externally peer reviewed. 